OPEN ACCESS

Edited by:

Anwen Shao,

Zhejiang University, China

Reviewed by:

Jianming Zhu,

Second Affiliated Hospital of Nanchang University, China

Sheng Tu,

Zhejiang University, China

*Correspondence:

Qian Li

liqianzhe2011@163.com

Specialty section:

This article was submitted to

Cellular Neuropathology,

a section of the journal

Frontiers in Cellular Neuroscience

Received: 19 December 2021

Accepted: 17 January 2022

Published: 11 February 2022

Citation:

Zhu S, Meng B, Jiang J, Wang $X$, Luo N, Liu N, Shen H, Wang L and

Li Q (2022) The Updated Role of Transcranial Ultrasound Neuromodulation in Ischemic Stroke: From Clinical and Basic Research. Front. Cell. Neurosci. 16:839023. doi: 10.3389/fncel.2022.839023

\section{The Updated Role of Transcranial Ultrasound Neuromodulation in Ischemic Stroke: From Clinical and Basic Research}

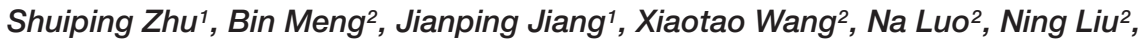 \\ Huaping Shen', Lu Wang ${ }^{3}$ and Qian Li ${ }^{2 *}$
}

${ }^{1}$ Department of Geriatric Medicine, Rongjun Hospital, Jiaxing, China, ${ }^{2}$ Department of Ultrasound, Rongjun Hospital, Jiaxing, China, ${ }^{3}$ Starbody Plastic Surgery Clinic, Hangzhou, China

Ischemic stroke is a common cause of death and disability worldwide, which leads to serious neurological and physical dysfunction and results in heavy economic and social burdens. For now, timely and effective dissolution of thrombus, and ultimately improvement in the recovery of neurological functions, is the treatment strategy focus. Recently, many studies have reported that transcranial ultrasound stimulation (TUS), as a non-invasive method, can dissolve thrombus, improve cerebral blood circulation, and exert a neuroprotective effect post-stroke. TUS can promote functional recovery and improve rehabilitation efficacy among patients with ischemic stroke. This mini-review summarizes the potential mechanism and limitation of TUS in stroke aims to provide a new strategy for the future treatment of patients with ischemic stroke.

Keywords: transcranial ultrasound stimulation, ischemic stroke, mechanism, review, limitation

\section{INTRODUCTION}

Ischemic stroke is the most common type of cerebrovascular disease. According to the data of the global burden of disease (GBD) study, stroke is the most common cause of death among Chinese residents (GBD 2019 Stroke Collaborators, 2021). From 2010 to 2019, the incidence of ischemic stroke has increased from $129 / 1,00,000$ in 2010 to $145 / 1,00,000$ in 2019 , and the prevalence of ischemic stroke has increased from $1,100 / 1,00,000$ in 2010 to 1,256/1,00,000 in 2019. According to China's aging population trend and the seventh census data, in 2021, there will be approximately 17.8 million patients with stroke, with 3.4 million new stroke patients and 2.3 million strokerelated deaths among the population over 40 years of age in China each year. China is the largest developing country, accounting for one-fifth of the world's total population, with the highest number of patients with stroke worldwide (Wang et al., 2017). Current treatments for ischemic stroke include thrombolysis, mechanical thrombectomy, and neuroprotective therapies (Liaw and Liebeskind, 2020). However, intravenous thrombolytic therapy has a strict treatment time window, with the risk of rebleeding, making its clinical application limited.

Statistical data have shown that only $16 \%$ of patients with acute ischemic stroke in China are admitted to the hospital within $3 \mathrm{~h}$ of symptom onset, and only $1.3 \%$ of these patients receive thrombolytic therapy (Wu et al., 2019; Shen et al., 2020; Tu et al., 2021a,b). Transcranial ultrasound stimulation (TUS) is a non-invasive technique for patients with stroke, which can stimulate specific brain areas, and improve neural activity and connectivity. The advantages of transcranial 
ultrasound for brain stimulation are that it does not necessitate surgery or genetic alteration but confers spatial resolutions superior to other non-invasive methods such as transcranial magnetic stimulation (TMS; Tufail et al., 2011; Kim et al., 2012). TMS uses magnetic fields to pass through the skull to stimulate the brain tissue and has been widely used for disorders caused by the brain lesions, such as those caused in the type of depression that does not respond to medication, and cognitive impairment after stroke. The disadvantage of TMS is the lack of good spatial resolution, which results in limitations in the application of neural rehabilitation (Dionísio et al., 2018; Krogh et al., 2021; Liu et al., 2021a). The characteristics of high penetration and high spatial resolution of TUS have shown therapeutic potential in stroke treatment (Guo et al., 2015; Li et al., 2017; Liu et al., 2019; Wu et al., 2020; Malinova et al., 2021). Transcranial ultrasound is roughly divided into two types based on frequency: one is diagnostic transcranial ultrasound, with a frequency of $1.0-15 \mathrm{MHz}$, whereas the other is transcranial aggregation ultrasound, with a frequency lower than 1.0 MHz (Yang et al., 2008; Deng et al., 2021). At present, most studies focus on the thrombolytic effect and the mechanism of neuroregulation in low-frequency TUS (Li et al., 2017; Fomenko et al., 2018; Liu et al., 2019). TUS is able to transmit a certain frequency of an ultrasonic wave on the human skull with a specific ultrasonic probe. Through the skull, the ultrasonic energy is transmitted to the brain tissues, stimulating the brain to produce a series of biological effects. TUS has been explored in arterial thrombolytic therapy and post-stroke rehabilitation therapy as an emerging and non-invasive brain stimulation method (Rubiera et al., 2008; Tsivgoulis et al., 2008; Barlinn et al., 2013).

\section{NEUROPROTECTIVE EFFECT OF TRANSCRANIAL ULTRASOUND STIMULATION IN STROKE}

The neuroprotective effect of TUS has become a hot topic in recent years, especially in the field of stroke. TUS mainly exerts a neuroprotective effect through the following mechanisms.

\section{Rapid Restoration of Cerebral Blood Supply and Improvement of Cerebral Blood Flow}

The earlier the restoration of cerebral blood supplies after acute cerebral infarction, the better the recovery of neurological function (Fisher and Bastan, 2008; Molina and AlvarezSabín, 2009). Therefore, improving cerebral circulation before irreversible changes occur in the brain tissue and alleviating neuronal damage are the key factors for the treatment of ischemic stroke and also have a positive significance in improving the rehabilitation efficacy post-stroke. After ischemic stroke, the neurons in the stroke center rapidly undergo apoptosis and necrosis because of ischemia and hypoxia, whereas the surrounding cells still have transient survival ability because of the existence of collateral circulation, thus forming an ischemic penumbra. Within a certain period of time, cells in this area can either undergo apoptosis or return to the normal brain tissue (Bonnin et al., 2021; Davis and Donnan, 2021; Yang and Liu, 2021). If the ischemic penumbra is rescued in time, it can effectively prevent stroke progression; further, the successful rescue of ischemic penumbra is also conducive to the recovery of the nervous system and physical function in the future. TUS has been proved to be beneficial for the improvement of cerebral blood circulation after acute ischemic stroke, and within a certain range, cerebral blood flow also shows a gradually increasing trend with the increase of stimulation intensity and duration (Yuan et al., 2020, 2021; Liu et al., 2021b). However, with an improvement in cerebral blood flow, the risk of rebleeding also increases. A previous clinical trial (Daffertshofer et al., 2005) has confirmed that low-frequency $(300 \mathrm{kHz})$ TUS not only resulted in improvement of the thrombolytic efficiency of tissue plasminogen activator (tPA) but also caused an increased rate of a cerebral hemorrhage in patients concomitantly treated with intravenous tPA. The limitation of the study was that only 26 patients were included. Combined lysis of thrombus with ultrasound and systemic tPA for emergent revascularization in acute ischemic stroke (CLOTBUST-ER), an international fourcenter phase II trial, demonstrated that in patients with acute ischemic stroke, transcranial ultrasound augments tPA-induced arterial recanalization with a non-significant trend toward an increased rate of clinical recovery from stroke, compared with the control group. The rates of symptomatic intracerebral hemorrhage were similar between the active and control groups (Schellinger et al., 2015; Katsanos et al., 2020). Further studies are still awaited on this important issue. A previous study has shown that the earlier the transcranial ultrasound intervention, the better the neuroprotective effect (Liu et al., 2019). Therefore, early use of TUS after stroke may effectively improve the brainblood supply, restore local blood circulation, rescue the ischemic penumbra, and ultimately reduce brain tissue damage.

Transcranial ultrasound stimulation has also been shown to improve the vascular recanalization rate, which is an important index to evaluate the treatment effect in acute ischemic stroke. Evgenii et al. (Kim et al., 2021) reported a wireless, wearable system to achieve ultrasound brain stimulation in freely behaving animals. The brain activity induced by the system was monitored as cerebral hemodynamic changes via near-infrared spectroscopy. The system was also applied to stroke rehabilitation after temporal middle cerebral artery occlusion (MCAO) in rats. The stimulation was found to induce hemodynamic changes in the sonicated area, whereas open-field tests showed that ultrasound applied to the ipsilateral hemisphere for 5 consecutive days after stroke facilitated recovery. Another study conducted by Wu et al. (2020) aimed to determine the neuroprotective effect of low-intensity TUS at different time points using endothelin1-induced MCAO in rats. The results showed that the rats that received low-intensity TUS exhibited reduced damage of the affected brain tissue after cerebral ischemia. The greatest protective effect was found with ultrasound stimulation of $30 \mathrm{~min}$ after cerebral ischemia. Hameroff et al. (2013) found that using $8 \mathrm{MHz}$ ultrasound to stimulate the upper frontotemporal cortex of patients for $15 \mathrm{~s}$ could increase arterial oxygen saturation, suggesting that ultrasound stimulation at a higher frequency may 
alleviate ischemic hypoxic changes after stroke. Furthermore, one study (Alexandrov et al., 2019) found that within $3 \mathrm{~h}$ after ischemic stroke onset, low-frequency transcranial ultrasound therapy within $30 \mathrm{~min}$ after thrombolysis can significantly enhance atenolol enzyme-induced arterial recanalization ability compared with the control group, but the results also indicated that transcranial ultrasound therapy did not significantly improve patient outcomes in 90 days after stroke.

\section{Reduced Inflammatory Response and Apoptosis}

Inflammatory mediators can cause further damage to neurons in the ischemic penumbra. A recent study applied low-intensity TUS to the ischemic cortex after distal MCAO and found that ultrasound may activate coagulation factors through certain signal transduction pathways and reduce neutrophils in the ischemic region, thus reducing the inflammatory response and facilitating neuronal recovery in the ischemic penumbra (Guo et al., 2015). Similarly, another research on Parkinson's disease rat model found that the low-intensity pulsed ultrasound treatment significantly inhibited 6-OHDA-induced glial activation and the phosphorylation of nuclear factor- $\kappa$ B p 65 in the substantia nigra pars compacta. Further evaluation revealed that low-intensity pulsed ultrasound effectively preserved the levels of neurotrophic factors, dopamine transporter, and tight junction proteins in the blood-brain barrier (Song et al., 2021). Furthermore, (Zhou et al., 2021) revealed that TUS reduced the chronic inflammatory response in microglia and astrocyte activation, whereas Pang et al. (2021) found that TUS could attenuate the level of TNF- $\alpha$.

As ischemic stroke induces cellular apoptosis, especially neuronal apoptosis, which leads to neurological dysfunction, determining a means to alleviate neuronal apoptosis has become an important issue for researchers while evaluating the outcome of patients with ischemic stroke. A study conducted in 2021 (Zhou et al., 2021) found that TUS could reduce the level of apoptosis-related protein Bax, and improved the movement and learning in aging rats. Su et al. (2017) further observed that low-intensity pulsed ultrasound could inhibit the progression of apoptosis following traumatic brain injury. Thus, the neuroprotective effects of TUS may be associated with the TrkB/Akt-CREB signaling pathway. Another study from the same team (Chen S. F. et al., 2018) found that the low-intensity pulsed ultrasound significantly attenuated the brain edema and neuronal death, reduced neutrophil infiltration and microglial activation, increased the $\mathrm{Bcl}-2 / \mathrm{Bax}$ ratio, and enhanced the phosphorylation of Bad and FOXO-1, ultimately improving the functional outcomes. These results indicated that the neuroprotective effects of TUS are associated with a reduction of early inflammatory events and inhibition of apoptotic progression.

\section{Promotion of the Release of Neurotrophic Factors}

Neurotrophic factors, such as brain-derived neurotrophic factor (BDNF) and glial cell line-derived neurotrophic factor (GDNF), are considered to be involved in the regulation of key nerve functions and neuroplasticity in stroke (Wang et al., 2006; Luo et al., 2019; Liu et al., 2020). A previous study has found that TUS effectively prevents cerebral ischemia/reperfusion injury through apoptosis reduction and BDNF induction (Chen C. M. et al., 2018). Su et al. (2017) observed that lowintensity pulsed ultrasound could increase the BDNF protein levels following a traumatic brain injury. The neuroprotective effects of TUS may be associated with the enhancement of protein levels of neurotrophic factors. Similarly, another study conducting MCAO using a C57BL/6J mouse model found that the low-intensity pulsed ultrasound accelerated the expression of BDNF in the brain of stroke mice and significantly moderated neuronal function after injury including neurological score, motor activity, and brain pathological score. In addition, Yang et al. (2015) demonstrate that TUS could enhance the protein levels of neurotrophic factors (i.e., BDNF, GDNF, VEGF, and GLUT1), which could have neuroprotective effects against neurodegenerative diseases. In a study using an ischemic stroke mouse model, low-intensity pulsed TUS could induce BDNF expression, decreasing the percentage of damaged neurons and the loss of neurological function after stroke. Tsai et al. (Zhang et al., 2019) interestingly found that TUS can also exert antidepressant-like effects. TUS could change the expression of BDNF in the hippocampus of rats. Given that BDNF plays an important role in the pathogenesis of depression, promoting BDNF could have a therapeutic effect (Hashimoto, 2010).

\section{Thrombolytic Effect of Transcranial Ultrasound Stimulation}

Ultrasound has direct and indirect thrombolytic effects. Indirect thrombolytic effects of TUS mainly enhance the effectiveness of thrombolytic drugs. In addition, ultrasound can directly dissolve the thrombus through its special physical and chemical properties (Cao et al., 2021; Doelare et al., 2021; Mei and Zhang, 2021). The main thrombolytic effects of TUS are summarized as follows.

\section{Cavitation Effect}

Cavitation is the generation of a large number of small bubbles when ultrasound is applied to a liquid (Lahiri et al., 2021). These bubbles rapidly vibrate, expand, and burst. A large amount of energy is released at the moment of bubble explosion, causing the thrombus to tear and decompose, exposing the surface of the thrombus in large quantities, and further accelerating the dissolution of the thrombus (Ma et al., 2020; Jo et al., 2021; Singh et al., 2021). The cavitation-induced thermal effect has been confirmed as a potential mechanism underlying this phenomenon. When a certain amount of heat is generated at the moment of bubble expansion, the heat effect may be the main role of ultrasound therapy, because a certain amount of heat effect can increase the activity of fibrinolytic enzymes, which is conducive to its binding with thrombosis (Wu et al., 2020). Intensity-focused ultrasound can generate high heat for the ablation of tumors and other biological tissues (ter Haar, 2007; Bessonova et al., 2010; Simon et al., 2012). The mechanism underlying this is complex. Some studies have proposed that high-intensity focused ultrasound can affect the action potential 


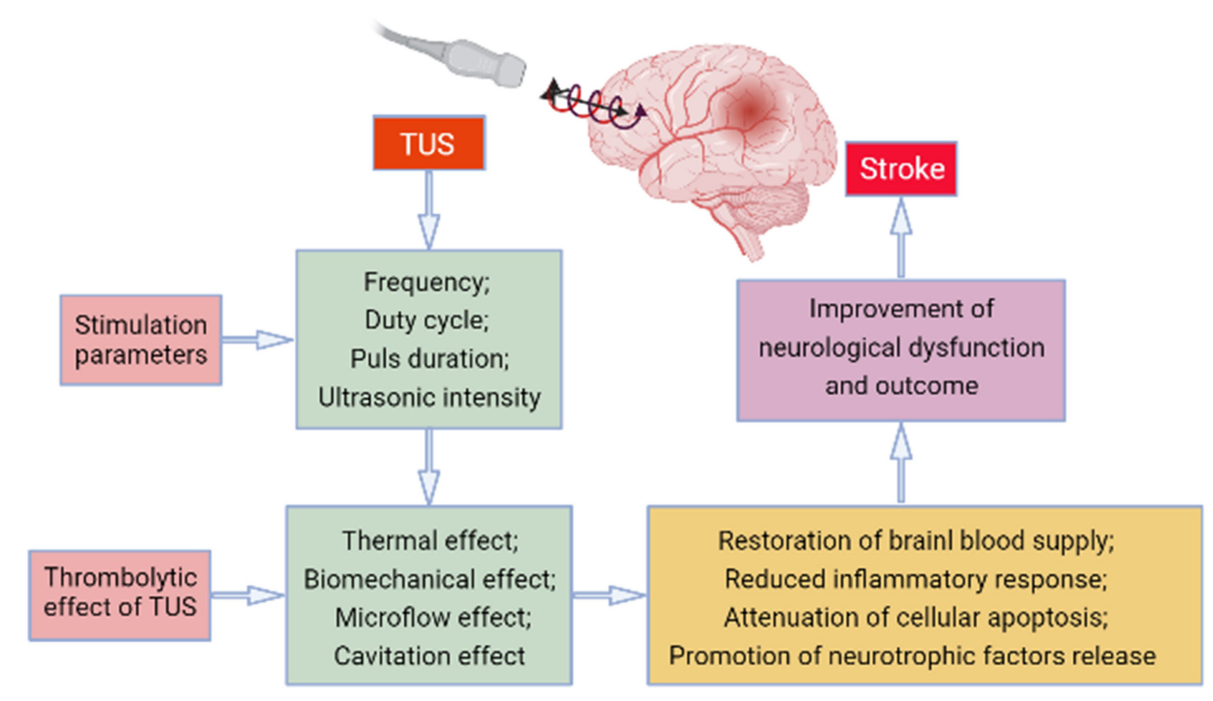

FIGURE 1 | The potential mechanism involved in the transcranial ultrasound stimulation (TUS)-induced effect on stroke.

of axons (Wahab et al., 2012); the electrical activity of neurons can be suppressed by disrupting the ultrastructure of synapses by blocking the connections between them (Borrelli et al., 1981; Tsui et al., 2005). Furthermore, the thermal effect of highfrequency and high-intensity focused ultrasound can damage nerve tissue and thus block transmission between synapses (Foley et al., 2008; Colucci et al., 2009). In contrast, one research found that although low-intensity TUS was eventually converted into heat energy when it passed through non-ideal media, the ultrasonic signal had almost no thermal effect on the brain tissue in the process of ultrasonic stimulation. The heat is extremely weak, much lower than the predicted heat required to produce obvious biological effects of temperature threshold (Liu et al., 2019). The differences in the abovementioned studies may be related to the differences between animal models and clinical patients. However, it is still controversial whether lowfrequency TUS has a meaningful thermal effect during treatment. Whether the strength of the thermal effect is related to the frequency, intensity, and duration of TUS, and whether the thermal effect will cause damage to the brain tissue, needs further research.

\section{Biomechanical Effect}

The mechanical action is the main mechanism of ultrasound thrombolysis. The mechanical vibration of ultrasound can destroy blood clots, decompose thrombi, increase the contact between enzyme and fibrin, and promote the dissolution of thrombi. Ultrasound can also enhance the vitality of the brain cells and promote the repair of nerve cells after cerebral ischemia (Masomi-Bornwasser et al., 2021; Shin Low et al., 2021).

\section{Microflow Effect}

The microflow effect is caused by the cavitation effect. Here, the pressure generated by the burst microbubble causes the liquid to form microflow, which can accelerate drugs to the ischemic region (Matsievskii, 2003; Park et al., 2019; Li et al., 2021).

\section{LIMITATIONS OF THE CURRENT TRANSCRANIAL ULTRASOUND STIMULATION STUDY}

Treatment with TUS lacks clinical trials, as there are great differences between animal models and clinical patients, many problems remain to be solved in the clinical application of TUS. Although some clinical studies have proved that TUS can improve the clinical efficacy in ischemic strokes, these evaluation methods cannot reflect the improvement in a patient's ability during the rehabilitation process. More detailed and specific assessment scales should be used for evaluating the effect of TUS, such as assessment of cognitive, speech, sensory, motor, and other aspects. Moreover, most of the current clinical studies are about a combination of TUS and thrombolytic drugs to improve the thrombolytic effect. Clinical research in the future may explore TUS and other rehabilitation treatment methods, such as physical therapy, speech therapy, and low-frequency neuromuscular electrical stimulation, to improve the effectiveness of rehabilitation therapy in patients with ischemic stroke. For example, anodal transcranial direct current stimulation combined with constraint-induced movement therapy resulted in the improvement of functional ability of the paretic upper limb compared with constraintinduced movement therapy alone, indicating that TUS may enhance the effect of rehabilitation in patients with chronic stroke (Figlewski et al., 2017). In addition, there is no clear standard answer with regard to the specific parameters of TUS. Previous studies found that transcranial ultrasound with different frequencies can produce different excitatory or inhibitory effects on the brain (Krishna et al., 2018; Zhang et al., 2021). The ultrasonic stimulation parameters mainly include frequency, pulse repetition frequency, duty cycle, pulse duration, and ultrasonic intensity (Uddin et al., 2021). Frequency refers to the number of oscillation cycles per unit time, importantly, as 
the frequency is inversely proportional to the wavelength, the higher the frequency, the smaller the focal spot volume, and the more significant the acoustic attenuation and scattering effect. Therefore, it is necessary to explore the most suitable frequency and intensity of TUS according to the location depth of the stimulation target and the thickness of penetration through the skull, and the safety of different parameter combinations in clinical application.

\section{CONCLUSION}

Ultrasound thrombolysis has already been used widely; low-frequency TUS can exert its effect based on the mechanical vibration, cavitation, or microflow, consequently dissolving the thrombus, reducing the infarct size in patients, promoting cerebral circulation, rescuing the ischemic penumbra, and improving the prognosis of patients with stroke. Low-frequency TUS is mostly used clinically at present. Numerous studies have explored the mechanism

\section{REFERENCES}

Alexandrov, A. V., Köhrmann, M., Soinne, L., Tsivgoulis, G., Barreto, A. D., Demchuk, A. M., et al. (2019). Safety and efficacy of sonothrombolysis for acute ischaemic stroke: a multicentre, double-blind, phase 3, randomised controlled trial. Lancet Neurol. 18, 338-347. doi: 10.1016/S1474-4422(19)30026-2

Barlinn, K., Tsivgoulis, G., Molina, C. A., Alexandrov, D. A., Schafer, M. E., Alleman, J., et al. (2013). Exploratory analysis of estimated acoustic peak rarefaction pressure, recanalization, and outcome in the transcranial ultrasound in clinical sonothrombolysis trial. J. Clin. Ultrasound 41, 354-360. doi: 10.1002/ jcu. 21978

Bessonova, O. V., Khokhlova, V. A., Canney, M. S., Bailey, M. R., and Crum, L. A. (2010). A derating method for therapeutic applications of high intensity focused ultrasound. Acoust. Phys. 56, 354-363. doi: 10.1134/s1063771010030140

Bonnin, P., Kubis, N., and Charriaut-Marlangue, C. (2021). Collateral supply in preclinical cerebral stroke models. Transl. Stroke Res. doi: 10.1007/s12975-02100969-3 [Epub ahead of print].

Borrelli, M. J., Bailey, K. I., and Dunn, F. (1981). Early ultrasonic effects upon mammalian CNS structures (chemical synapses). J. Acoust. Soc. Am. 69, 15141516. doi: $10.1121 / 1.385791$

Cao, W., Liu, Y., Ran, P., He, J., Xie, S., Weng, J., et al. (2021). Ultrasound-propelled janus rod-shaped micromotors for site-specific sonodynamic thrombolysis. ACS Appl. Mater. Interf. 13, 58411-58421. doi: 10.1021/acsami.1c19288

Chen, C. M., Wu, C. T., Yang, T. H., Liu, S. H., and Yang, F. Y. (2018). Preventive effect of low intensity pulsed ultrasound against experimental cerebral ischemia/reperfusion injury via apoptosis reduction and brain-derived neurotrophic factor induction. Sci. Rep. 8:5568. doi: 10.1038/s41598-01823929-8

Chen, S. F., Su, W. S., Wu, C. H., Lan, T. H., and Yang, F. Y. (2018). Transcranial ultrasound stimulation improves long-term functional outcomes and protects against brain damage in traumatic brain injury. Mol. Neurobiol. 55, 7079-7089. doi: 10.1007/s12035-018-0897-z

Colucci, V., Strichartz, G., Jolesz, F., Vykhodtseva, N., and Hynynen, K. (2009). Focused ultrasound effects on nerve action potential in vitro. Ultrasound Med. Biol. 35, 1737-1747. doi: 10.1016/j.ultrasmedbio.2009.05.002

Daffertshofer, M., Gass, A., Ringleb, P., Sitzer, M., Sliwka, U., Els, T., et al. (2005). Transcranial low-frequency ultrasound-mediated thrombolysis in brain ischemia: increased risk of hemorrhage with combined ultrasound and tissue plasminogen activator: results of a phase II clinical trial. Stroke 36, 1441-1446. doi: 10.1161/01.STR.0000170707.86793.1a

Davis, S. M., and Donnan, G. A. (2021). Ischemic penumbra: a personal view. Cerebrovasc. Dis. 50, 656-665. doi: 10.1159/000519730 of low-frequency TUS and confirmed that low-frequency TUS has certain neuroregulatory effects, which have both excitatory and inhibitory effects on the human cerebral cortex (Figure 1).

In summary, although there are still many issues that need to be explored and solved in terms of clinical application, numerous studies have shown that TUS can promote thrombolysis, increase cerebral blood circulation, and improve neurological recovery. Thus, after ischemic stroke onset, timely and proper TUS intervention may improve the neurological function and quality of life of post-stroke patients.

\section{AUTHOR CONTRIBUTIONS}

QL participated in the design of the review. SZ, BM, JJ, XW, $\mathrm{NLu}$, and NLi drafted the manuscript. HS and LW critically revised the text and figure. All authors read and approved the final manuscript.

Deng, L., Yang, S. D., O’Reilly, M. A., Jones, R. M., and Hynynen, K. (2021) An ultrasound-guided hemispherical phased array for microbubble-mediated ultrasound therapy. IEEE Trans. Biomed. Eng. doi: 10.1109/TBME.2021. 3132014 [Epub ahead of print].

Dionísio, A., Duarte, I. C., Patrício, M., and Castelo-Branco, M. (2018). The use of repetitive transcranial magnetic stimulation for stroke rehabilitation: a systematic review. J. Stroke Cerebrovasc. Dis. 27, 1-31. doi: 10.1016/j. jstrokecerebrovasdis.2017.09.008

Doelare, S. A. N., Jean Pierre, D. M., Nederhoed, J. H., Smorenburg, S. P. M., Lely, R. J., Jongkind, V., et al. (2021). Microbubbles and ultrasound accelerated thrombolysis for peripheral arterial occlusions: the outcomes of a single arm phase II trial. Eur. J. Vasc. Endovasc. Surg. 62, 463-468. doi: 10.1016/j.ejvs.2021. 05.030

Figlewski, K., Blicher, J. U., Mortensen, J., Severinsen, K. E., Nielsen, J. F., and Andersen, H. (2017). Transcranial direct current stimulation potentiates improvements in functional ability in patients with chronic stroke receiving constraint-induced movement therapy. Stroke 48, 229-232. doi: 10.1161/ STROKEAHA.116.014988

Fisher, M., and Bastan, B. (2008). Treating acute ischemic stroke. Curr. Opin. Drug Discov. Dev. 11, 626-632.

Foley, J. L., Little, J. W., and Vaezy, S. (2008). Effects of high-intensity focused ultrasound on nerve conduction. Muscle Nerve 37, 241-250. doi: 10.1002/mus. 20932

Fomenko, A., Neudorfer, C., Dallapiazza, R. F., Kalia, S. K., and Lozano, A. M. (2018). Low-intensity ultrasound neuromodulation: an overview of mechanisms and emerging human applications. Brain Stimul. 11, 1209-1217. doi: 10.1016/j.brs.2018.08.013

GBD 2019 Stroke Collaborators (2021). Global, regional, and national burden of stroke and its risk factors, 1990-2019: a systematic analysis for the Global Burden of Disease Study 2019. Lancet Neurol. 20, 795-820. doi: 10.1016/S14744422(21)00252-0

Guo, T., Li, H., Lv, Y., Lu, H., Niu, J., Sun, J., et al. (2015). Pulsed transcranial ultrasound stimulation immediately after the ischemic brain injury is neuroprotective. IEEE Trans. Biomed. Eng. 62, 2352-2357. doi: 10.1109/ TBME.2015.2427339

Hameroff, S., Trakas, M., Duffield, C., Annabi, E., Gerace, M. B., Boyle, P., et al. (2013). Transcranial ultrasound (TUS) effects on mental states: a pilot study. Brain Stimul. 6, 409-415. doi: 10.1016/j.brs.2012.05.002

Hashimoto, K. (2010). Brain-derived neurotrophic factor as a biomarker for mood disorders: an historical overview and future directions. Psychiatry Clin. Neurosci. 64, 341-357. doi: 10.1111/j.1440-1819.2010.0 2113.x 
Jo, J., Forrest, M. L., and Yang, X. (2021). Ultrasound-assisted laser thrombolysis with endovascular laser and high-intensity focused ultrasound. Med. Phys. 48, 579-586. doi: 10.1002/mp.14636

Katsanos, A. H., Alexandrov, A. V., Mandava, P., Köhrmann, M., Soinne, L., Barreto, A. D., et al. (2020). Pulse pressure variability is associated with unfavorable outcomes in acute ischaemic stroke patients treated with intravenous thrombolysis. Eur. J. Neurol. 27, 2453-2462. doi: 10.1111/ene. 14447

Kim, E., Anguluan, E., Kum, J., Sanchez-Casanova, J., Park, T. Y., Kim, J. G., et al. (2021). Wearable transcranial ultrasound system for remote stimulation of freely moving animal. IEEE Trans. Biomed. Eng. 68, 2195-2202. doi: 10.1109/ TBME.2020.3038018

Kim, H., Taghados, S. J., Fischer, K., Maeng, L. S., Park, S., and Yoo, S. S. (2012). Noninvasive transcranial stimulation of rat abducens nerve by focused ultrasound. Ultrasound Med. Biol. 38, 1568-1575. doi: 10.1016/j.ultrasmedbio. 2012.04.023

Krishna, V., Sammartino, F., and Rezai, A. (2018). A review of the current therapies, challenges, and future directions of transcranial focused ultrasound technology: advances in diagnosis and treatment. JAMA Neurol. 75, 246-254. doi: 10.1001/jamaneurol.2017.3129

Krogh, S., Jønsson, A. B., Aagaard, P., and Kasch, H. (2021). Efficacy of repetitive transcranial magnetic stimulation for improving lower limb function in individuals with neurological disorders: a systematic review and meta-analysis of randomized sham-controlled trials. J. Rehabil. Med. doi: 10.2340/jrm.v53. 1097 [Epub ahead of print].

Lahiri, S., Mandal, D., Biswas, S., Gogate, P. R., and Bhardwaj, R. L. (2021). Sonocatalytic recovery of ceria from graphite and inhibition of graphite erosion by ionic liquid based platinum nanocatalyst. Ultrason. Sonochem. 82:105863. doi: 10.1016/j.ultsonch.2021.105863

Li, H., Sun, J., Zhang, D., Omire-Mayor, D., Lewin, P. A., and Tong, S. (2017). Low-intensity $(400 \mathrm{~mW} / \mathrm{cm}(2), 500 \mathrm{kHz})$ pulsed transcranial ultrasound preconditioning may mitigate focal cerebral ischemia in rats. Brain Stimul. 10, 695-702. doi: 10.1016/j.brs.2017.02.008

Li, Y., Wu, J., Fu, L., and Wang, J. (2021). A fast bubble detection method in microtubes based on pulsed ultrasound. Micromachines (Basel) 12:1402. doi: $10.3390 / \mathrm{mi1} 2111402$

Liaw, N., and Liebeskind, D. (2020). Emerging therapies in acute ischemic stroke. F1000Res 9:F1000FacultyRev-546.

Liu, L., Du, J., Zheng, T., Hu, S., Dong, Y., Du, D., et al. (2019). Protective effect of low-intensity transcranial ultrasound stimulation after differing delay following an acute ischemic stroke. Brain Res. Bull. 146, 22-27. doi: 10.1016/j.brainresbull. 2018.12.004

Liu, W., Wang, X., O’Connor, M., Wang, G., and Han, F. (2020). Brain-derived neurotrophic factor and its potential therapeutic role in stroke comorbidities. Neural. Plast. 2020:1969482. doi: 10.1155/2020/1969482

Liu, Y., Li, H., Zhang, J., Zhao, Q. Q., Mei, H. N., and Ma, J. (2021a). A meta-analysis: whether repetitive transcranial magnetic stimulation improves dysfunction caused by stroke with lower limb spasticity. Evid. Based Complement Alternat. Med. 2021:7219293. doi: 10.1155/2021/7219293

Liu, Y., Wang, G., Cao, C., Zhang, G., Tanzi, E. B., Zhang, Y., et al. (2021b). Neuromodulation effect of very low intensity transcranial ultrasound stimulation on multiple nuclei in rat brain. Front. Aging Neurosci. 13:656430. doi: 10.3389/fnagi.2021.656430

Luo, W., Liu, T., Li, S., Wen, H., Zhou, F., Zafonte, R., et al. (2019). The serum BDNF level offers minimum predictive value for motor function recovery after stroke. Transl. Stroke Res. 10, 342-351. doi: 10.1007/s12975-018-0648-5

Ma, L., Wang, Y., Zhang, S., Qian, X., Xue, N., Jiang, Z., et al. (2020). Deep penetration of targeted nanobubbles enhanced cavitation effect on thrombolytic capacity. Bioconjug. Chem. 31, 369-374. doi: 10.1021/acs.bioconjchem.9b 00653

Malinova, V., Bleuel, K., Stadelmann, C., Iliev, B., Tsogkas, I., Psychogios, M. N., et al. (2021). The impact of transcranial direct current stimulation on cerebral vasospasm in a rat model of subarachnoid hemorrhage. J. Cereb. Blood Flow Metab. 41, 2000-2009. doi: 10.1177/0271678X21990130

Masomi-Bornwasser, J., Fabrig, O., Krenzlin, H., König, J., Tanyildizi, Y., Kempski, O., et al. (2021). Systematic analysis of combined thrombolysis using ultrasound and different fibrinolytic drugs in an in vitro clot model of intracerebral hemorrhage. Ultrasound Med. Biol. 47, 1334-1342. doi: 10.1016/j.ultrasmedbio. 2021.01.005

Matsievskii, D. D. (2003). Ultrasound as a tool in experimental research of macro- and microcirculation. Bull. Exp. Biol. Med. 136, 101-104. doi: 10.1023/a: 1026065602829

Mei, L., and Zhang, Z. (2021). Advances in biological application of and research on low-frequency ultrasound. Ultrasound Med. Biol. 47, 2839-2852. doi: 10.1016/j. ultrasmedbio.2021.06.005

Molina, C. A., and Alvarez-Sabín, J. (2009). Recanalization and reperfusion therapies for acute ischemic stroke. Cerebrovasc. Dis. 27(Suppl. 1), 162-167.

Pang, N., Huang, X., Zhou, H., Xia, X., Liu, X., Wang, Y., et al. (2021). Transcranial ultrasound stimulation of hypothalamus in aging mice. IEEE Trans. Ultrason. Ferroelectr. Freq. Control 68, 29-37. doi: 10.1109/TUFFC.2020.2968479

Park, A. Y., Kwon, M., Woo, O. H., Cho, K. R., Park, E. K., Cha, S. H., et al. (2019). A prospective study on the value of ultrasound microflow assessment to distinguish malignant from benign solid breast masses: association between ultrasound parameters and histologic microvessel densities. Korean J. Radiol. 20, 759-772. doi: 10.3348/kjr.2018.0515

Rubiera, M., Ribo, M., Delgado-Mederos, R., Santamarina, E., Maisterra, O., Delgado, P., et al. (2008). Do bubble characteristics affect recanalization in stroke patients treated with microbubble-enhanced sonothrombolysis? Ultrasound Med. Biol. 34, 1573-1577. doi: 10.1016/j.ultrasmedbio.2008.02.011

Schellinger, P. D., Alexandrov, A. V., Barreto, A. D., Demchuk, A. M., Tsivgoulis, G., Kohrmann, M., et al. (2015). Combined lysis of thrombus with ultrasound and systemic tissue plasminogen activator for emergent revascularization in acute ischemic stroke (CLOTBUST-ER): design and methodology of a multinational phase 3 trial. Int. J. Stroke 10, 1141-1148. doi: 10.1111/ijs. 12536

Shen, Y., Chao, B. H., Cao, L., Tu, W. J., and Wang, L. D. (2020). Stroke center care and outcome: results from the CSPPC stroke program. Transl. Stroke Res. 11, 377-386. doi: 10.1007/s12975-019-00727-6

Shin Low, S., Nong Lim, C., Yew, M., Siong Chai, W., Low, L. E., Manickam, S., et al. (2021). Recent ultrasound advancements for the manipulation of nanobiomaterials and nanoformulations for drug delivery. Ultrason. Sonochem. 80:105805. doi: 10.1016/j.ultsonch.2021.105805

Simon, J., Sapozhnikov, O., Khokhlova, V., Wang, Y. N., Crum, L., and Bailey, M. (2012). Tissue atomization by high intensity focused ultrasound. IEEE Int. Ultrason. Symp. 2012, 1003-1006.

Singh, R., Jo, J., Riegel, M., Forrest, M. L., and Yang, X. (2021). The feasibility of ultrasound-assisted endovascular laser thrombolysis in an acute rabbit thrombosis model. Med. Phys. 48, 4128-4138. doi: 10.1002/mp.15068

Song, W. S., Sung, C. Y., Ke, C. H., and Yang, F. Y. (2021). Anti-inflammatory and neuroprotective effects of transcranial ultrasound stimulation on Parkinson's disease. Ultrasound Med. Biol. 48, 265-274. doi: 10.1016/j.ultrasmedbio.2021. 10.001

Su, W. S., Wu, C. H., Chen, S. F., and Yang, F. Y. (2017). Transcranial ultrasound stimulation promotes brain-derived neurotrophic factor and reduces apoptosis in a mouse model of traumatic brain injury. Brain Stimul. 10, 1032-1041. doi: 10.1016/j.brs.2017.09.003

ter Haar, G. (2007). Therapeutic applications of ultrasound. Prog. Biophys. Mol. Biol. 93, 111-129.

Tsivgoulis, G., Culp, W. C., and Alexandrov, A. V. (2008). Ultrasound enhanced thrombolysis in acute arterial ischemia. Ultrasonics 48, 303-311. doi: 10.1016/j. ultras.2007.11.008

Tsui, P. H., Wang, S. H., and Huang, C. C. (2005). In vitro effects of ultrasound with different energies on the conduction properties of neural tissue. Ultrasonics 43 , 560-565. doi: 10.1016/j.ultras.2004.12.003

Tu, W. J., Chao, B. H., Ma, L., Yan, F., Cao, L., Qiu, H., et al. (2021a). Casefatality, disability and recurrence rates after first-ever stroke: a study from bigdata observatory platform for stroke of China. Brain Res. Bull. 175, 130-135. doi: 10.1016/j.brainresbull.2021.07.020

Tu, W. J., Yan, F., Chao, B. H., Ma, L., Ji, X. M., and Wang, L. D. (2021b). Thrombolytic DNT and fatality and disability rates in acute ischemic stroke: a study from bigdata observatory platform for stroke of China. Neurol. Sci. 43, 677-682. doi: 10.1007/s10072-021-05580-w

Tufail, Y., Yoshihiro, A., Pati, S., Li, M. M., and Tyler, W. J. (2011). Ultrasonic neuromodulation by brain stimulation with transcranial ultrasound. Nat. Protoc. 6, 1453-1470. doi: 10.1038/nprot.2011.371 
Uddin, S. M. Z., Komatsu, D. E., Motyka, T., and Petterson, S. (2021). Low-intensity continuous ultrasound therapies - a systematic review of current state-of-theart and future perspectives. J. Clin. Med. 10:2698. doi: 10.3390/jcm10122698

Wahab, R. A., Choi, M., Liu, Y., Krauthamer, V., Zderic, V., and Myers, M. R. (2012). Mechanical bioeffects of pulsed high intensity focused ultrasound on a simple neural model. Med. Phys. 39, 4274-4283. doi: 10.1118/1.4729712

Wang, W., Jiang, B., Sun, H., Ru, X., Sun, D., Wang, L., et al. (2017). Prevalence, incidence, and mortality of stroke in China: results from a nationwide population-based survey of 480 ?687 adults. Circulation 135, 759-771. doi: 10 . 1161/CIRCULATIONAHA.116.025250

Wang, Y., Alexander, O. B., Woodward-Pu, Y. M., Stahl, C. E., and Borlongan, C. V. (2006). Viral vector strategy for glial cell line-derived neurotrophic factor therapy for stroke. Front. Biosci. 11:1101-1107. doi: 10.2741/1866

Wu, S., Wu, B., Liu, M., Chen, Z., Wang, W., Anderson, C. S., et al. (2019). Stroke in China: advances and challenges in epidemiology, prevention, and management. Lancet Neurol. 18, 394-405. doi: 10.1016/S1474-4422(18)30500-3

Wu, S., Zheng, T., Du, J., Yuan, Y., Shi, Q., Wang, Z., et al. (2020). Neuroprotective effect of low-intensity transcranial ultrasound stimulation in endothelin-1induced middle cerebral artery occlusion in rats. Brain Res. Bull. 161, 127-135. doi: 10.1016/j.brainresbull.2020.05.006

Yang, F. Y., Fu, W. M., Chen, W. S., Yeh, W. L., and Lin, W. L. (2008). Quantitative evaluation of the use of microbubbles with transcranial focused ultrasound on blood-brain-barrier disruption. Ultrason. Sonochem. 15, 636-643. doi: 10.1016/ j.ultsonch.2007.08.003

Yang, F. Y., Lu, W. W., Lin, W. T., Chang, C. W., and Huang, S. L. (2015). Enhancement of neurotrophic factors in astrocyte for neuroprotective effects in brain disorders using low-intensity pulsed ultrasound stimulation. Brain Stimul. 8, 465-473. doi: 10.1016/j.brs.2014.11.017

Yang, S. H., and Liu, R. (2021). Four decades of ischemic penumbra and its implication for ischemic stroke. Transl. Stroke Res. 12, 937-945. doi: 10.1007/ s12975-021-00916-2

Yuan, Y., Wang, Z., Liu, M., and Shoham, S. (2020). Cortical hemodynamic responses induced by low-intensity transcranial ultrasound stimulation of mouse cortex. Neuroimage 211:116597. doi: 10.1016/j.neuroimage.2020.116597
Yuan, Y., Zhang, K., Zhang, Y., Yan, J., Wang, Z., Wang, X., et al. (2021). The effect of low-intensity transcranial ultrasound stimulation on neural oscillation and hemodynamics in the mouse visual cortex depends on anesthesia level and ultrasound intensity. IEEE Trans. Biomed. Eng. 68, 1619-1626. doi: 10.1109/ TBME.2021.3050797

Zhang, D., Li, H., Sun, J., Hu, W., Jin, W., Li, S., et al. (2019). Antidepressant-like effect of low-intensity transcranial ultrasound stimulation. IEEE Trans. Biomed. Eng. 66, 411-420. doi: 10.1109/TBME.2018.2845689

Zhang, T. T., Pan, N., Wang, Y. P., Liu, C. Y., and Hu, S. M. (2021). Transcranial focused ultrasound neuromodulation: a review of the excitatory and inhibitory effects on brain activity in human and animals. Front. Hum. Neurosci. 15:749162. doi: 10.3389/fnhum.2021.749162

Zhou, H., Meng, L., Xia, X., Lin, Z., Zhou, W., Pang, N., et al. (2021). Transcranial ultrasound stimulation suppresses neuroinflammation in a chronic mouse model of Parkinson's disease. IEEE Trans. Biomed. Eng. 68, 3375-3387. doi: 10.1109/TBME.2021.3071807

Conflict of Interest: The authors declare that the research was conducted in the absence of any commercial or financial relationships that could be construed as a potential conflict of interest.

Publisher's Note: All claims expressed in this article are solely those of the authors and do not necessarily represent those of their affiliated organizations, or those of the publisher, the editors and the reviewers. Any product that may be evaluated in this article, or claim that may be made by its manufacturer, is not guaranteed or endorsed by the publisher.

Copyright (c) 2022 Zhu, Meng, Jiang, Wang, Luo, Liu, Shen, Wang and Li. This is an open-access article distributed under the terms of the Creative Commons Attribution License (CC BY). The use, distribution or reproduction in other forums is permitted, provided the original author(s) and the copyright owner(s) are credited and that the original publication in this journal is cited, in accordance with accepted academic practice. No use, distribution or reproduction is permitted which does not comply with these terms. 